Determination of saturation, heart rate, and respiratory rate at forearm using a Nellcor (TM) forehead $\mathrm{SpO}(2)$-saturation sensor

\title{
Harju, Jarkko
}

2017-10

Harju , J , Vehkaoja , A , Lindroos , V , Kumpulainen, P, Liuhanen , S , Yli-Hankala , A \& Oksala, N 2017 , ' Determination of saturation, heart rate, and respiratory rate at forearm using a Nellcor (TM) forehead $\mathrm{SpO}(2)$-saturation sensor ' , Journal of Clinical Monitoring and Computing , vol. 31 , no. 5 , pp. 1019-1026 . https://doi.org/10.1007/s10877-016-9940-7

http://hdl.handle.net/10138/245625

https://doi.org/10.1007/s10877-016-9940-7

publishedVersion

Downloaded from Helda, University of Helsinki institutional repository.

This is an electronic reprint of the original article.

This reprint may differ from the original in pagination and typographic detail.

Please cite the original version. 


\title{
Determination of saturation, heart rate, and respiratory rate at forearm using a Nellcor ${ }^{\mathrm{TM}}$ forehead $\mathrm{SpO}_{2}$-saturation sensor
}

\author{
Jarkko Harju $^{1}$ - Antti Vehkaoja ${ }^{2}$ - Ville Lindroos ${ }^{3}$ - Pekka Kumpulainen ${ }^{2}$. \\ Sasu Liuhanen ${ }^{4} \cdot$ Arvi Yli-Hankala ${ }^{1,3} \cdot$ Niku Oksala $^{3,5}$
}

Received: 18 April 2016/Accepted: 7 October 2016/Published online: 17 October 2016

(C) Springer Science+Business Media Dordrecht 2016

\begin{abstract}
Alterations in arterial blood oxygen saturation, heart rate (HR), and respiratory rate (RR) are strongly associated with intra-hospital cardiac arrests and resuscitations. A wireless, easy-to-use, and comfortable method for monitoring these important clinical signs would be highly useful. We investigated whether the Nellcor ${ }^{\mathrm{TM}}$ OxiMask MAX-FAST forehead sensor could provide data for vital sign measurements when located at the distal forearm instead of its intended location at the forehead to provide improved comfortability and easy placement. In a prospective setting, we recruited 30 patients undergoing surgery requiring postoperative care. At the postoperative care unit, patients were monitored for two hours using a standard patient monitor and with a study device equipped with a Nellcor ${ }^{\mathrm{TM}}$ Forehead $\mathrm{SpO}_{2}$ sensor. The readings were electronically recorded and compared in post hoc analysis using Bland-Altman plots, Spearman's correlation, and root-mean-square error (RMSE). Bland-Altman plot showed that saturation $\left(\mathrm{SpO}_{2}\right)$ differed by a mean of $-0.2 \%$ points (SD, 4.6), with a patient-weighted Spear-
\end{abstract}

Jarkko Harju

Jarkko.harju@fimnet.fi

1 Department of Anesthesia, Tampere University Hospital, PL2000, 33521 Tampere, Finland

2 Tampere University of Technology, Tampere, Finland

3 Medical School, University of Tampere, Tampere, Finland

4 Department of Anesthesia, Helsinki University Hospital, Helsinki, Finland

5 Department of Surgery, Tampere University Hospital, Tampere, Finland man's correlation ( $r$ ) of 0.142 , and an RMSE of 4.2 points. For HR measurements, the mean difference was $0.6 \mathrm{bpm}$ $(\mathrm{SD}, 2.5), r=0.997$, and RMSE $=1.8$. For RR, the mean difference was $-0.51 / \mathrm{min}$ (4.1), $r=0.586$, and $\mathrm{RMSE}=4.0$. The $\mathrm{SpO}_{2}$ readings showed a low mean difference, but also a low correlation and high RMSE, indicating that the Nellcor ${ }^{\mathrm{TM}}$ saturation sensor cannot reliably assess oxygen saturation at the forearm when compared to finger PPG measurements.

Keywords Intraoperative monitoring · Plethysmography · Pulse oximetry $\cdot$ Heart rate $\cdot$ Respiratory rate

\section{Introduction}

Worsening vital signs are strongly associated with intrahospital cardiac arrest and resuscitation [1]. The clinical signs most commonly associated with patient deterioration are arterial blood oxygen saturation $\left(\mathrm{SpO}_{2}\right)$, heart rate (HR), respiratory rate (RR), and blood pressure [2]. There is presently no single device capable of wirelessly monitoring all of these parameters, although several reports describe attempts to develop such a device [3-5].

The Nellcor ${ }^{\mathrm{TM}}$ Forehead $\mathrm{SpO}_{2}$ sensor is a reflectance mode photoplethysmography (PPG) sensor that was developed to measure $\mathrm{SpO}_{2}$ and $\mathrm{HR}$ at the forehead [6-9]. Studies of the saturation measurement of the use of this sensor on the forehead report it to be accurate, with measurements comparable to those acquired using traditional transmittance mode sensors on the finger [6,9]. Saturation and HR have been measured successfully at wrist and palmar region in infants using sensors based on light absorbance. $[10,11]$ However, in adults the skin thickness is too great to reach accurate measurements. One previous 
study described the performance of saturation sensors on the wrist area [10], but to our knowledge Nellcor forehead sensor has not been tested on distal forearm. Based on our pilot measurements, distal forearm region showed promising results for saturation measurement. Another study compared the power spectrum of PPG recordings at different sites, reporting the greatest power at the fingers for HR and at the forearm for RR [12]. The HR signal was also present at distal forearm. Combinining this information, distal forearm region might have potential to monitor saturation, HR and RR using just a single sensor.

In clinical monitoring, RR is typically estimated using the impedance pneumography from electrocardiogram (ECG) leads. However, the measurement of electrical impedance has marked limitations associated with chest wall movements. Multiple factors, such as incorrect electrode placement, coughing, and crying, may generate a high number of inaccurate readings $[13,14]$. The respiration-associated alterations on PPG waveform remain partly unknown [15], but multiple changes in the waveform caused by RR have been described. [16-19] RR measurement using PPG has been compared to thoracic impedance under postoperative care conditions, showing promising results, with a low proportion of false-positive breath recordings $(4.6 \pm 4.5 \%)$ [20]. More recent studies have reported similar results [21-23].

$\mathrm{SpO}_{2}$ and $\mathrm{HR}$ are typically measured at the finger, forehead, or ear lobe. While sensor fixation represents a marked challenge, the use of a wristband at distal forearm could offer an easier and more comfortable site for the sensors. Here we investigated off-label use of the Nellcor $^{\mathrm{TM}}$ PPG sensor at a previously unreported location-the distal forearm. The device was also equipped with an accelerometer to evaluate whether accuracy could be improved by excluding measurements taken during movement. This feasibility study aimed to assess whether a readily available sensor could reliably wirelessly monitor the common clinical signs of $\mathrm{SpO}_{2}, \mathrm{HR}$, and RR at a single location.

\section{Materials and methods}

This observational study included 30 patients (mean age of $67[\mathrm{SD}=13]$ years), who were recruited between January and May 2015 at Tampere University Hospital. Each collection peried lasted for two hours during post-operative care treatment. The patients were undergoing elective surgery requiring invasive blood pressure measurement. Patients with an implanted cardiac pacemaker were excluded. All patients gave their written informed consent prior to study entry, and the study was approved by the local ethics committee (ETL R13145).

\subsection{Monitoring}

Standard care included patient monitoring with a GE Carescape $^{\mathrm{TM}}$ B650 monitor (GE Healthcare Oy, Helsinki, Finland) using a TruSignal ${ }^{\mathrm{TM}}$ saturation sensor on the finger. Recordings from this monitor were collected at 10-s intervals using S5 Collect ${ }^{\mathrm{TM}}$ software (GE Healthcare Oy, Helsinki, Finland) for all of the study parameters. $\mathrm{SpO}_{2}$ and HR measurements from the standard monitor were based on finger measurements, while RR was based on impedance measurement from the ECG electrodes.

In addition to standard monitoring, patients were equipped with a study device mounted in a single unit using a rechargeable battery, which was wrapped around the forearm with a flexible band. The study device comprised a Nellcor ${ }^{\mathrm{TM}}$ OxiMask MAX-FAST forehead sensor for $\mathrm{SpO}_{2}$ (Covidien, MN, USA) along with a 3-D accelerometer (Freescale MMA8452Q; NXP, Eindhoven, Netherlands). A Faros ECG recorder (Mega Electronics, Oulu, Finland) was used to obtain a three-lead ECG to allow additional synchronizing. The study device sensor was placed over the radial bone at the distal forearm near the wrist, and correct placement was indicated by an LED controlled by the signal-processing unit of the $\mathrm{SpO}_{2}$ sensor. Optimal placement was determined using pre-clinical voluntary pilot measurements, which indicated that placement over the radial bone enabled the strongest signal. The study device recorded $\mathrm{SpO}_{2}, \mathrm{HR}$, and RR every $10 \mathrm{~s}$, and accelerometer data at $10-\mathrm{Hz}$ frequencies. The data were sent via Wi-Fi connection to an Internet remote server, and the data were viewed online on a web page and obtained electronically after measurements.

The RR was obtained from the PPG sensor at the forearm using a novel algorithm (Oksala $\mathrm{N}$ and Liuhanen $\mathrm{S}$, Patent WO2015107268 A1) [24]. Our present report is the first to describe the performance of this algorithm. Briefly, the algorithm extracts a good quality beat series by removing normal noise, and then interpolating missing beats and deleting erroneously detected ones. From the original signal, the algorithm extracts four primary subsignals and two derived sub-signals, each of which is independently analyzed in both time and frequency domains. The changes in the PPG waveform that were used consisted of baseline modulation, amplitude modulation, respiratory sinus arrhythmia and pulse wave width. Finally, good quality estimates are combined to obtain a RR estimate.

The values obtained from the standard monitor and the study device were synced using the local time shown on the Internet web page. To allow additional syncing, the arterial line was occluded and the ECG connection from the Faros simultaneously removed. An independent observer (J.H. 
and V.L.) recorded all device and patient movements, and verified the data collection accuracy.

In order to compare the test monitor the PPG-derived RR was additionally compared against a validation data described by Charlton et al. [19], which is commonly available online for study purposes [25]. The RR from the dataset was recalculated using our algorithm [24] and compared against impedance measurement provided along the data.

The accelerometer data were used to test whether measurement accuracy could be improved by excluding the readings obtained during movement. We used the summation value for three-dimensional movement. Thereafter, the threshold for movement detection was formed by defining a mean value and standard deviation for the lowest $90 \%$ of the summation of the acceleration data. We calculated the mean plus three times the standard deviation for use as a threshold for "slight movement", and the mean plus ten times the standard deviation for use as the threshold for "definite movement". This limit definition was performed using a modification of a commonly used technique for detection limit determination [26].

\subsection{Power analysis}

The power analysis was based on the results of a previous study that compared measurements of reflectance and transmittance mode saturation sensors at the forehead and fingers, reporting a bias difference of -1.39 compared to -2.61 units (SD, 1.3) [9]. Assuming similar bias differences in the present study, we would need 19 patients to achieve a power of $0.80(p<0.05)$. To account for the possibility of patient drop-out, we recruited 30 patients.

\subsection{Statistical analysis}

Data were analyzed using IBM SPSS statistics version 23 (IBM, IL, USA) and with the method described in a previous publication [27] using Microsoft Excel 2010 (Microsoft, Redmond, WA, USA) for Bland-Altman Plot. Results are reported using the method described by Bland and Altman for multiple comparisons [28]. To determine the bias of the novel method compared to traditional measurement, we calculated the Spearman's correlation between the devices and the root-mean-square error (RMSE) to describe the mean error. The correlation between datapoints was calculated patient-weighted as a correlation between each patients values. The sensor comparison data were found to be scattered, and are reported as median and interquartile range (IQR; $25-75$ th percentile). The patient demographics are reported as mean (SD) as for normally distributed data. The $p$ value was calculated using the Wilcoxon signed-rank test, or using the Mann-Whitney U or Kruskal-Wallis test for the RMSE. $p$ value $<0.05$ was considered statistically significant and RMSE $>4.0$ clinically unacceptable.

\section{Results}

Of the 30 patients studied, 13 had peripheral arterial disease. In two patients, the recording was unsuccessful due to a breakdown of the sensor cable during measurement, leaving 28 patients for final analysis. There were a total of $10,767 \mathrm{SpO}_{2}, 14,832 \mathrm{HR}$ and $18,857 \mathrm{RR}$ pairs left for comparisons. Table 1 describes the patients' characteristics. The following types of surgery were performed prior to measurements: vascular surgery in 13 patients $(46.4 \%)$, gastroenterological surgery in 8 patients $(28.6 \%)$, urologic surgery in 2 patients $(7.1 \%)$, plastic surgery in 3 patients $(10.7 \%)$, and orthopedic surgery in 2 patients $(7.1 \%)$. Patients were predominantly lying in the supine position.

In the Bland-Altman plot, we found a low mean difference in $\mathrm{SpO}_{2}$ [ $-0.3 \%$ points $(95 \%$ confidence interval -3.9 to 5.1), Limits of agreement (LoA) $)_{\text {upper: }} 7.2$ (CI 6.1 to 8.1), $\mathrm{LoA}_{\text {lower }}-7.9$ (CI -8.8 to -6.8$)$ ] with a low patientweighted Spearman's correlation between devices $(r=0.142)$. The HR showed a low mean difference [0.6 bpm (CI -0.85 to 2.05), $\mathrm{LoA}_{\text {upper }} 5.6$ (CI 5.2 to 5.9). $\mathrm{LoA}_{\text {lower }}-4.4(\mathrm{CI}-4.8$ to -4.0$\left.)\right]$ and a good correlation $(r=0.997)$. RR differed by -0.6 cycles (CI -3.9 to 5.1 , $\mathrm{LoA}_{\text {upper }} 8.0$ (CI 7.2 to 8.6 ), $\mathrm{LoA}_{\text {lower }}-6.8$ (CI -7.4 to $-6.0)$, with a moderate correlation $(r=0.586)$. The RMSE comparison was 4.2 points for $\mathrm{SpO}_{2}, 4.0$ points for $\mathrm{RR}$, and 1.8 points for HR (Fig. 1; Table 2).

There were a total 25,154 data comparisons for 39 subjects in validation data set. The method comparison in validation data for RR revealed a bias of 4 breaths (CI 4.0 to 4.2), while the RMSE comparison was 5.6 (Table 2). RR differed by 4.1 breaths (CI -2.7 to 10.8), LoA $_{\text {upper }} 11.9$ (CI 11.1 to 12.7$)$, $\mathrm{LoA}_{\text {lower }}-3.8$ (CI -3.0 to -4.7 ) as described in Fig. 2.

Table 1 Patient characteristics, described as mean (SD) or frequency $(\%)$

\begin{tabular}{ll}
\hline Characteristics & $n=30$ \\
\hline Age (years) & $67(13)$ \\
Gender F/M & $13 / 17(43 \% / 57 \%)$ \\
Height $(\mathrm{cm})$ & $172(10)$ \\
Weight $(\mathrm{kg})$ & $80(16)$ \\
ASA I/II/III/IV & $1 / 7 / 21 / 1$ \\
Peripheral artery disease & $13(43 \%)$ \\
Atrial fibrillation & $3(10 \%)$ \\
Coronary artery disease & $1(3 \%)$ \\
Forearm circumference $(\mathrm{cm})$ & $18(2)$ \\
\hline
\end{tabular}



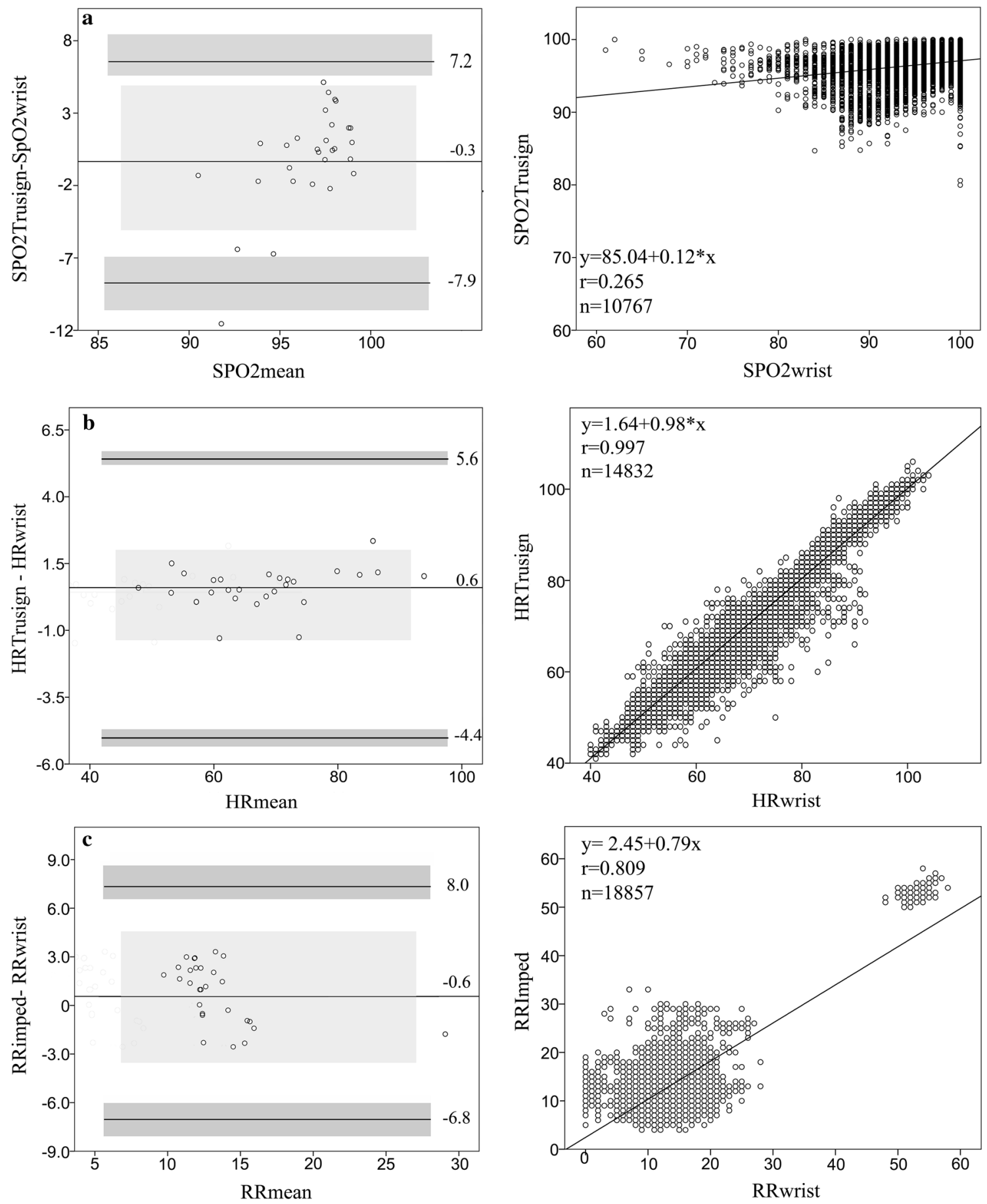

Fig. 1 Bland-Altman plot and Spearman correlation for saturation (a), heart rate (b) and respiratory rate (c). The data is presented with a Bland-Altman plot (BA) and Spearman correlation between all

Slight movement was detected during $12.6 \%$ of the total recording time. Movement was associated with higher RMSE in all tested parameters. For $\mathrm{SpO}_{2}$, the difference was significant $(p=0.037)$ but of a small magnitude (Table 3). RMSE did not differ in association with peripheral arterial disease, body mass index (BMI), or forearm circumference.

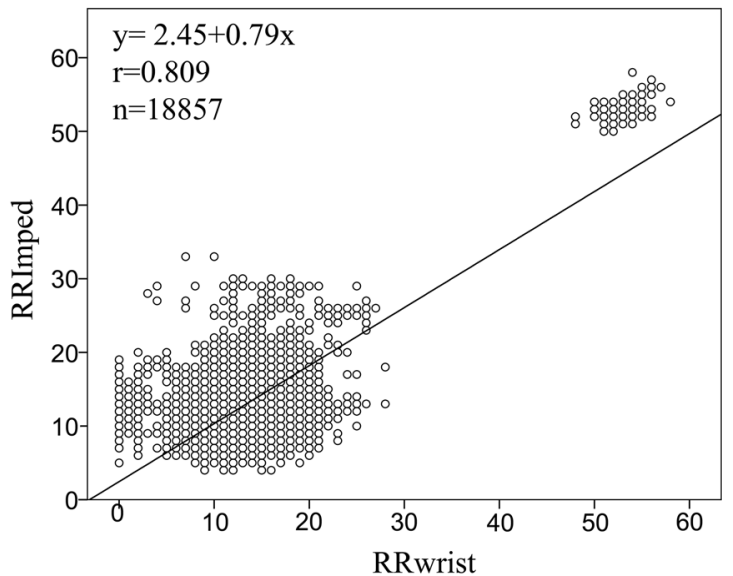

measurements. In BA plot black lines represent mean and limits of agreement, grey areas describe the $95 \%$ confidence intervals respectively

\section{Discussion}

The present study investigated the performance of a readily available PPG sensor for measuring common clinical signs at a single location. Our main finding was that the forehead PPG sensor could not reliably detect $\mathrm{SpO}_{2}$ at the forearm when compared to finger PPG recordings. In particular, the 
Table 2 Patient-weighted value described as median (IQR) and as root-mean-square error (RMSE) (IQR), Spearman correlation and $p$ value for the difference between test device and reference monitor $(\mathrm{B} 650)(n=28)$ or validation data. $(n=38)$

\begin{tabular}{lllllllr}
\hline & Plethysmography & Min, max & References & Min, max & RMSE & Spearman correlation & $p$ \\
\hline $\mathrm{SPO}_{2}(\%)$ & $97.5(95.6-99.2)$ & 86,100 & $97.0(95.5-97.8)$ & 91,100 & $4.2(2.8-5.7)$ & 0.142 & 0.399 \\
$\mathrm{HR}(\mathrm{bpm})$ & $67.7(60.0-73.2)$ & 48,94 & $67.6(59.4-73.9)$ & 47,93 & $1.8(1.6-2.9)$ & 0.997 & 0.001 \\
$\mathrm{RR}(1 / \mathrm{min})$ & $13.2(12.3-14.4)$ & 11,28 & $12.1(10.5-14.1)$ & 9,30 & $4.0(3.1-4.7)$ & 0.586 & 0.048 \\
RR validation & $12.1(10.7-14.0)$ & 9,19 & $16.5(13.6-19.0)$ & 7,26 & $5.6(1.7-6.8)$ & 0.416 & $<0.001$ \\
\hline
\end{tabular}
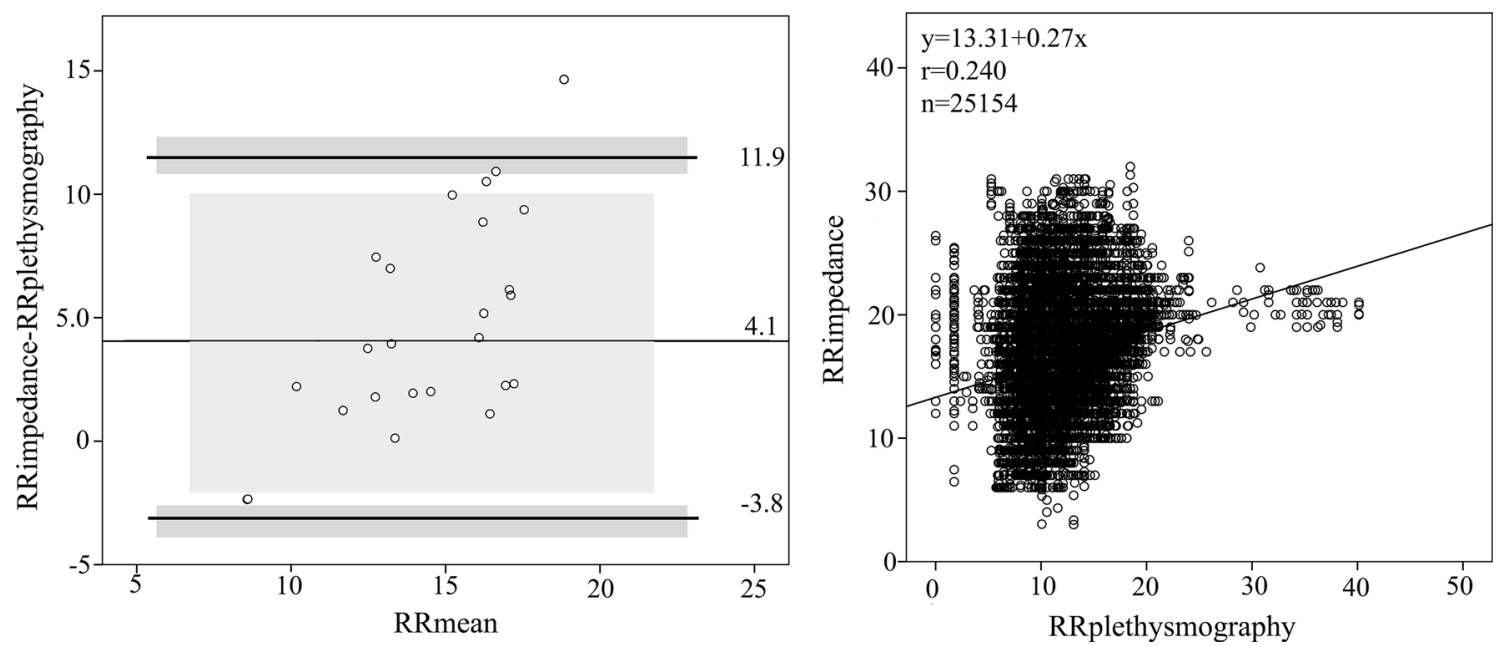

Fig. 2 Bland-Altman plot and Spearman correlation for HR in validation data. The data is presented with a Bland-Altman plot (BA) for multiple measurements and Spearman correlation between all

RMSE was outside of the $2 \%$ accuracy range required by a previous recommendation [29] and $4.0 \%$ recommended by ISO standard [30]. Respiratory rate measurements showed a moderate patient-weighted correlation, and a moderately high RMSE, and Bland-Altman plots of HR recordings showed a small mean difference and a very strong correlation between the devices. Thus, our findings indicated that HR could be reliably measured by a PPG sensor at the forearm. The accuracy of RR, measured at forearm, was moderate. The performance of $\mathrm{SpO}_{2}$ was poor.

\subsection{Saturation}

Our results showed that when placed at forearm instead of intended location at forehead, oximetry measurements were inaccurate compared to finger oximetry measurements. Previous evaluation of the accuracy of finger sensor oximetry reported by the manufacturer revealed an RMSE of \pm 2 ( \pm 3 during motion). The mean difference in our present study was small, but the correlation between the devices was poor and the RMSE describing the error in forehead sensor measurement was unacceptably high. The Nellcor $^{\mathrm{TM}}$ OxiMask MAX-FAST sensor detects the reflection of light, and several reports describe its efficacy, measurements. In BA plot black lines represent mean and limits of agreement, grey areas describe the $95 \%$ confidence intervals respectively

especially when placed on the forehead $[6,23,31]$. Here, we instead placed the sensor over the radial bone, a surface that is more rounded and potentially more prone to movement. Moreover, in the forearm location, there is a much longer distance between the device and the reflecting bone due to fatty tissue. Our results showed that RMSE did not differ in relation to movement, BMI, or forearm circumference.

The gold standard method for monitoring $\mathrm{SpO}_{2}$ is arterial oxygen saturation; however, this requires invasive measurements [29]. Motion artefacts and hypoperfusion are the most common causes of inaccurate $\mathrm{SpO}_{2}$ measurement at the fingers [32]. We detected a small but significant change in accuracy associated with movement. During critical illness, centralization of blood circulation decreases the accuracy of the $\mathrm{SpO}_{2}$ measurement at the fingers [6]. As all of our patients were electively treated, very few $\mathrm{SpO}_{2}$ readings were below $90 \%$.

There was a low correlation and high RMSE found in our study. The fingers are sensitive to mild hypothermia [33], which could result in a lower $\mathrm{SpO}_{2}$ in the fingers compared to in the distal forearm area. During our study measurements, the forearm and the device were constantly visible, which could have influenced the temperature in 


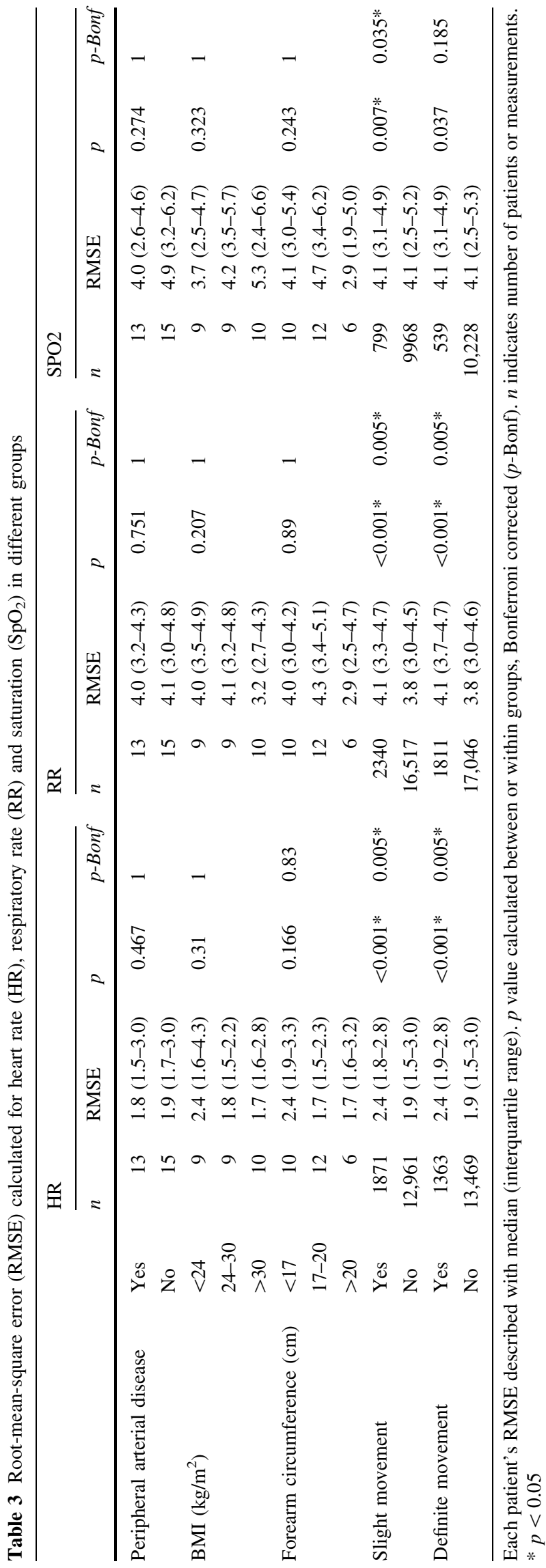

fingers. Unfortunately, we were unable to measure arterial oxygen saturation, which could have shed more light on this subject.

\subsection{Heart rate}

HR measurement was found to be very accurate, with a high correlation between the devices. This is in line with previous findings suggesting good accuracy [3-5]. Indeed, the RMSE for HR measurement was constantly low despite all confounding factors. Movement was the only factor that significantly affected HR measurement and the difference between the devices was still low. Previous studies evaluating wrist-based optical HR monitors have focused on fitness devices used by young healthy people, and there exists no prior data in hospital patients.

\subsection{Respiratory rate}

Our RR findings showed a good accuracy and moderate patient-weighted correlation between the devices. These findings are in line with those of Nilsson et al. [12] who reported that the distal forearm area is a suitable location for RR and HR measurement, although they found that spectral power was lower for HR than for RR. Another previous study compared RR calculated from finger PPG signal with RR from capnogram monitoring [21], and reported difference similar to that found in our study. Fingers are highly susceptible to movement, making measurement more difficult in mobile patients. Our patients were mostly lying, and slight or stronger movement of the forearm was detected during only $12.6 \%$ of the measurement period.

The comparison of the study algorithm against validation data [19] showed worse performance than against our patient data as decribed in Table 2. There might be several reasons for this difference. Our study data is recorded from distal forearm region where the RR signal has a greater power range [12]. The validation data patients were also moving which increases the diffulty in measuring the RR adequately. Furthermore, in the validation data, the plethysmographic waveform was collected using finger sensor based on absorption, thus the anatomic site and used sensor technique were different to our own measurements.

Estimating RR from a peripheral location is a complex task. As with $\mathrm{SpO}_{2}$, typical obstacles to RR measurement include movement artifacts and signal amplitude due to low perfusion $[15,19]$. The peripheral arterial disease may also affect the measurement, although our study could not demonstrate the difference. It is often seen that upper extremity arteries remain intact, although lower extremity arteries are seriously affected by peripheral artery disease. [34] Here we were able to achieve the reported accuracy by 
utilizing a strategy that treats different features of the PPG signal as independent components, and by continuously selecting which ones to use based on their quality.

\subsection{Limitations}

The present study has several limitations. Firstly, the inclusion of only patients who were undergoing invasive blood pressure measurement selected patients who had many comorbidities, and were undergoing major surgery with a high operation risk. However, the recordings were taken at a postoperative care unit under relatively stable conditions; thus, the findings are likely not specific to conditions of low $\mathrm{SpO}_{2}$ or low blood perfusion. Additionally, all patients were continuously observed, and study personnel screened the signal quality throughout the monitoring period. Secondly, the patients showed relatively stable hemodynamics during the study period, and were lying in a supine position. Therefore, our findings may not be generalized to patients with hypotension or delirium. Moreover, all patients had an incremental supply of oxygen. Thirdly, the recordings were acquired using two different devices and were synchronized in post hoc-analysis and, thus, we were unable to perform a beat-to-beat comparison. However, the recordings were taken over a short time-period, and were started and ended at the same time with both devices, decreasing the likelihood of a major desynchronization bias. Lastly the comparison to validation data was performed at a post hoc analysis and the site of measurement was different than that used in the present study Therefore the value of the comparison to validation data is limited.

\section{Conclusions}

Only a limited selection of currently available devices are capable of wirelessly monitoring vital signs [35, 36], and none can collect all commonly monitored clinical signs from a single site. Here we report that, compared to recordings from a finger sensor, $\mathrm{SpO}_{2}$ measurement at the wrist showed a poor correlation and a high RMSE. With regards to $R R$, the mean difference in the Bland-Altman plot was small and Spearman's correlation was moderate, but the RMSE was markedly high. Also the RMSE was higher in the validation data. The study device's measurement of HR showed good accuracy and correlation. To our knowledge, no commercially available sensors indicated for medical use can detect $\mathrm{SpO}_{2}$ at the wrist. The development of such a device might provide a reliable way to measure common clinical signs at one location.
Acknowledgments The authors wish to thank the Paulo Foundation, Finnish Society of Anaesthesiologists and the Finnish Cultural Foundation, Pirkanmaa Regional Fund for grants, as well as the Medieta Oy (Helsinki, Finland), which provided the study device.

\section{Compliance with ethical standards}

Conflicts of interest SL and NO have a pending patent on RR measurement. NO has been a shareholder on a former company Medieta. AV and PK have been former employees on a former company Medieta. JH, VL, and AY-H declare no conflicts of interest.

Ethical approval This study was approved by the Pirkanmaa Hospital district ethics committee. All procedures involving human participants were performed in accordance with the ethical standards of the institutional and/or national research committee, as well as with the 1964 Helsinki declaration and its later amendments or comparable ethical standards.

\section{References}

1. Schein RM, Hazday N, Pena M, Ruben BH, Sprung CL. Clinical antecedents to in-hospital cardiopulmonary arrest. Chest. 1990;98:1388-92.

2. Jones DA, DeVita MA, Bellomo R. Rapid-response teams. N Engl J Med. 2011;365:139-46.

3. Fukushima H, Kawanaka H, Bhuiyan MS, Oguri K. Estimating heart rate using wrist-type Photoplethysmography and acceleration sensor while running. Conf Proc IEEE Eng Med Biol Soc. 2012;2012:2901-4.

4. Renevey P, Sola J, Theurillat P, Bertschi M, Krauss J, Andries D, Sartori C. Validation of a wrist monitor for accurate estimation of RR intervals during sleep. Conf Proc IEEE Eng Med Biol Soc. 2013;2013:5493-6.

5. Zhang Z, Pi Z, Liu B. TROIKA: a general framework for heart rate monitoring using wrist-type photoplethysmographic signals during intensive physical exercise. IEEE Trans Biomed Eng. 2015;62:522-31.

6. Nesseler N, Frenel JV, Launey Y, Morcet J, Malledant Y, Seguin P. Pulse oximetry and high-dose vasopressors: a comparison between forehead reflectance and finger transmission sensors. Intensive Care Med. 2012;38:1718-22.

7. Sugino S, Kanaya N, Mizuuchi M, Nakayama M, Namiki A. Forehead is as sensitive as finger pulse oximetry during general anesthesia. Can J Anaesth. 2004;51:432-6.

8. Agashe GS, Coakley J, Mannheimer PD. Forehead pulse oximetry: headband use helps alleviate false low readings likely related to venous pulsation artifact. Anesthesiology. 2006;105:1111-6.

9. Schallom L, Sona C, McSweeney M, Mazuski J. Comparison of forehead and digit oximetry in surgical/trauma patients at risk for decreased peripheral perfusion. Heart Lung. 2007;36:188-94.

10. Safar H, El-Dash H. Pulse oximetry: Could wrist and ankle be alternative placement sites? Clin Pediatr (Phila). 2015;54:1375-9.

11. Phattraprayoon N, Sardesai S, Durand M, Ramanathan R. Accuracy of pulse oximeter readings from probe placement on newborn wrist and ankle. J Perinatol. 2012;32:276-80.

12. Nilsson L, Goscinski T, Kalman S, Lindberg LG, Johansson A. Combined photoplethysmographic monitoring of respiration rate and pulse: a comparison between different measurement sites in spontaneously breathing subjects. Acta Anaesthesiol Scand. 2007;51:1250-7. 
13. Wiklund L, Hok B, Stahl K, Jordeby-Jonsson A. Postanesthesia monitoring revisited: frequency of true and false alarms from different monitoring devices. J Clin Anesth. 1994;6:182-8.

14. Gaucher A, Frasca D, Mimoz O, Debaene B. Accuracy of respiratory rate monitoring by capnometry using the Capnomask $(\mathrm{R})$ in extubated patients receiving supplemental oxygen after surgery. Br J Anaesth. 2012;1(08):316-20.

15. Meredith DJ, Clifton D, Charlton P, Brooks J, Pugh CW, Tarassenko L. Photoplethysmographic derivation of respiratory rate: a review of relevant physiology. J Med Eng Technol. 2012;36:1-7.

16. Johansson A, Öberg PÅ. Estimation of respiratory volumes from the photoplethysmographic signal. Part I: experimental results. Med Biol Eng Comput. 1999;37:42-7.

17. Dash S, Shelley KH, Silverman DG, Chon KH. Estimation of respiratory rate from ECG, photoplethysmogram, and piezoelectric pulse transducer signals: a comparative study of timefrequency methods. IEEE Trans Biomed Eng. 2010;57:1099-107.

18. Lazaro J, Gil E, Bailon R, Minchole A, Laguna P. Deriving respiration from photoplethysmographic pulse width. Med Biol Eng Comput. 2013;51:233-42.

19. Charlton PH, Bonnici T, Tarassenko L, Clifton DA, Beale R, Watkinson PJ. An assessment of algorithms to estimate respiratory rate from the electrocardiogram and photoplethysmogram. Physiol Meas. 2016;37:610-26.

20. Nilsson L, Johansson A, Kalman S. Monitoring of respiratory rate in postoperative care using a new photoplethysmographic technique. J Clin Monit Comput. 2000;16:309-15.

21. Addison PS, Watson JN, Mestek ML, Ochs JP, Uribe AA, Bergese SD. Pulse oximetry-derived respiratory rate in general care floor patients. J Clin Monit Comput. 2015;29:113-20.

22. Garde A, Karlen W, Ansermino JM, Dumont GA. Estimating respiratory and heart rates from the correntropy spectral density of the photoplethysmogram. PLoS ONE. 2014;9:e86427.

23. Nilsson L, Johansson A, Kalman S. Respiration can be monitored by photoplethysmography with high sensitivity and specificity regardless of anaesthesia and ventilatory mode. Acta Anaesthesiol Scand. 2005;49:1157-62.

24. Oksala N, Liuhanen S (2015) Method and device for the detection of respiratory rate. PCT/FI2015/050023.

25. Charlton PH (2016) In: Synthetic dataset: dataset for verification of algorhitm implementions. Charlton Peter H. http://peterh charlton.github.io/RRest/synthetic_dataset.html. Accessed 10 Aug 2016.
26. Shrivastava A, Gupta V. Methods for the determination of limit of detection and limit of quantitation of the analytical methods. Chron Young Sci. 2011;2:21-5.

27. Zou GY. Confidence interval estimation for the Bland-Altman limits of agreement with multiple observations per individual. Stat Methods Med Res. 2013;22:630-42.

28. Bland JM, Altman DG. Agreement between methods of measurement with multiple observations per individual. J Biopharm Stat. 2007;17:571-82.

29. Batchelder PB, Raley DM. Maximizing the laboratory setting for testing devices and understanding statistical output in pulse oximetry. Anesth Analg. 2007;105:S85-94.

30. Finnish Standards association (2011) Medical electrical equipment-Part 2-61: particular requirements for basic safety and essential performance of pulse oximeter equipment (ISO 80601-2-61:2011).

31. Fischer MO, Fornier W, Hanouz JL, Fellahi JL. Cephalic and digital pulse oximetry in cardiac surgery: a comparative pilot study with arterial oximetry. Eur J Anaesthesiol. 2015;32:60-1.

32. Salyer JW. Neonatal and pediatric pulse oximetry. Respir Care. 2003;48:386-96 (discussion 397-8).

33. MacLeod DB, Cortinez LI, Keifer JC, Cameron D, Wright DR, White WD, Moretti EW, Radulescu LR, Somma J. The desaturation response time of finger pulse oximeters during mild hypothermia. Anaesthesia. 2005;60:65-71.

34. Tendera M, Aboyans V, Bartelink M, Baumgartner I, Clement D, Collet J, Cremonesi A, De Carlo M, Erbel R, Fowkes FG, Heras M, Kownator S, Minar E, Ostergren J, Poldermans D, Riambau V, Roffi M, Röther J, Sievert H, van Sambeek M, Zeller T. ESC Guidelines on the diagnosis and treatment of peripheral artery diseases. Eur Heart J. 2011;32:2851-906.

35. Hernandez-Silveira M, Ahmed K, Ang SS, Zandari F, Mehta T, Weir R, Burdett A, Toumazou C, Brett SJ. Assessment of the feasibility of an ultra-low power, wireless digital patch for the continuous ambulatory monitoring of vital signs. BMJ Open. 2015;5:e006606.

36. Nair D, Tan SY, Gan HW, Lim SF, Tan J, Zhu M, Gao H, Chua $\mathrm{NH}$, Peh WL, Mak KH. The use of ambulatory tonometric radial arterial wave capture to measure ambulatory blood pressure: the validation of a novel wrist-bound device in adults. J Hum Hypertens. 2008;22:220-2. 